\title{
An Initial Study On The Construction Of Ground Truth Binarized Images Of Ancient Palm Leaf Manuscripts
}

\author{
Made Windu Antara Kesiman, Sophea Prum, Jean-Christophe Burie, Jean-Marc Ogier \\ Laboratoire Informatique Image Interaction (L3i) \\ University of La Rochelle, Avenue Michel Crépeau, 17042 \\ La Rochelle Cedex 1, France \\ \{made_windu_antara.kesiman, sophea.prum, jcburie, jean-marc.ogier\}@univ-lr.fr
}

\begin{abstract}
Ancient palm leaf manuscripts are one of the very valuable cultural heritages that store various forms of knowledge and historical records of social life in Southeast Asia. The automatic analysis of these documents, in order to extract relevant information, is a real challenge. However, to evaluate the developed extraction algorithms, a ground truth is absolutely necessary. In this paper, we present some of the challenges of the state of the art binarization methods as an initial study for the construction of ground truth binarized images of palm leaf manuscripts. We propose and analyze the need for a specific scheme for the construction of the ground truth of binarized images. The aim of this scheme is to achieve a better ground truth for low quality palm leaf manuscripts. We experimentally tested and evaluated our proposed specific scheme and got promising results. This scheme adapts and performs better in constructing the ground truth of binarized images for palm leaf manuscripts.
\end{abstract}

Keywords—ground truth construction; binarization; low quality document image; palm leaf manuscript

\section{INTRODUCTION}

The existence of ancient palm leaf manuscripts in Southeast Asia is very important both in term of quantity and variety of historical content. In the last five years, ancient palm leaf manuscripts have received great attention from researchers in the field of document image analysis. For example, a digitization project for palm leaf manuscripts from Cambodia ${ }^{1}$ and Thailand [1], [2].

The ancient palm leaf manuscript is written on a dried palm leaf by using some sort of sharp pen or small knife, which is then scrubbed with natural dyes. Due to these specific characteristics, ancient palm leaf manuscripts are providing new challenges in document analysis.

Binarization process as one of the early and important stage document analysis pipeline, is also a real challenge for ancient palm leaf manuscripts. In order to evaluate and to select an optimal binarization method, the ground truth binarized image is necessary. Unfortunately, based on our knowledge, there is no existing public ground truth binarized image of palm leaf manuscripts. Therefore, creating a new ground truth binarized

\footnotetext{
${ }^{1}$ http://www.khmermanuscripts.org/
}

image of palm leaf manuscripts is a necessary step in our research.

Manual creation ground truth of binarized images (e.g. with PixLabeler application [3]) is a time consuming task. Therefore, in the research domain, several semi-automatic frameworks for the construction of ground truth binarized images have been presented [4]-[7] to reduce the time of ground truthing process. The human intervention is required only for some necessary tasks. For the DIBCO competition series [8], the ground truth binarized images are constructed using a semi-automatic procedure described in [5]. This procedure is an improved version of the previous work proposed in [4] which used an initial binarized image to obtain the skeleton of the characters, and a dilation process constrained by the edges of the character to construct the ground truth binarized images. This procedure is adapted and improved by some other works on the construction of ground truth binarized images. For instance, in [9], a similar method is used to create ground truth of a large document databases. In [6], in order to save user time in manual modification process by expert, two features of phase congruency are used to preprocess Persian heritage images to generate a rough initial binarized image. In [7], the ground truth binarized image of machine-printed document is constructed by segmenting and clustering the characters during the foreground enhancement step. User can manually add and remove character model assignments to degraded character instances. Unfortunately, it is impossible to validate a ground truth construction methodology to create a perfect ground truth image from a real image. The ground truth images are normally accepted based on visual observation.

In this paper, we analyse the previous works on construction of ground truth binarized images, especially based on the method proposed and used for DIBCO competition series. The purpose of our work is to achieve a better ground truth binarized images for low quality palm leaf manuscripts.

This paper is organized as follow: section 2 briefly presents the construction of ground truth framework presented in [4] and [5], and draw some remarks and propositions for the construction of ground truth of palm leaf manuscripts. Section 3 addresses the challenges in binarization process of palm leaf manuscript images and describes the specific scheme proposed for the initial binarization process for the construction of ground truth binarized images of palm leaf manuscripts. The 
experimental results are presented in Section 4 and conclusions with some prospects for the future work are given in Section 5.

\section{STUDY OF EXISTING FRAMEWORKS FOR CONSTRUCTION OF GROUND TRUTH BINARIZED IMAGES}

The construction of ground truth binarized images proposed in [4], consists of several steps: initial binarization process, skeletonization of the characters, manual correction of skeleton, and second skeletonization after manual correction process. The estimated ground truth image is then constructed by dilating the corrected skeleton image, constrained by the character edges (detected using Canny algorithm [10]) and the binarized image under evaluation. The skeleton is dilated until half of the Canny edges intersect each binarized component. The detail algorithm in pseudo code can be found in [4]. In this method, poor quality of initial binarized image will directly affect the result of the estimated ground truth. The ground truth image constructed strongly depends on the binarized image used as a constraint during the dilation process of the skeleton. The ground truth binarized images used for the DIBCO competition series are constructed with a modified procedure [5] as illustrated in Fig. 1. In this procedure, the conditional dilation step of the skeleton is constrained only by Canny edge image, without any initial binarized image.

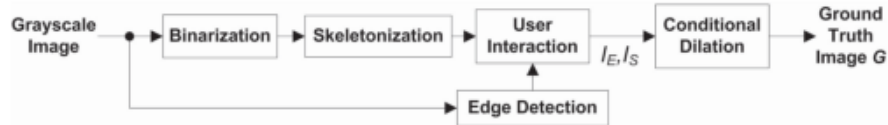

Fig. 1. Ground truth construction procedure used for DIBCO series [5]

We implemented the construction of ground truth binarized image based on the procedure proposed in [4] and the modified version in [5]. Based on our preliminary experiments, it is expected to obtain a good initial binarized image as the input to the next process of ground truth creation. The initial binarization method used in the stage of construction of skeletonized ground truth image should be able to generate an optimal and acceptable 'good enough' skeleton which detects and keeps the form of the characters. Image of skeleton generated in this step will facilitate the manual correction process. The more we have a correct skeleton the more the manual process is easier and faster.

After some experiments, we notice that for a nondegraded palm leaf manuscript, a simple global thresholded binarization method is sufficient to generate an acceptable binarized image and optimal image of skeleton. However, this method is not adapted to degraded palm leaf manuscripts. Fig. 2 shows some examples of skeletonized image generated with Matlab standard function bwmorph ${ }^{2}$ from different binarized images using different binarization methods. Influenced by the dried palm leaf texture, the stroke of characters in palm leaf manuscripts is thickened and widened. As a consequence, a lot of small short unuseful branchs on the skeleton are generated. Because of the poor quality of binarized and skeletonized image, the step of manual correction of the skeleton is very time consuming, it takes almost 8 hours for only one image of palm leaf manuscript. Therefore, in the case of degraded and low quality palm leaf manuscript images, our initial study

${ }^{2}$ http://fr.mathworks.com/help/images/ref/bwmorph.html focused on the development of an initial binarization process for the construction of ground truth binarized images (see Section III). One other important remark, superposing image of skeleton on the original image to guide the manual correction process is not enough. A priori knowledge of the form of ancient characters is mandatory to guarantee that the incomplete character skeleton can be completed in a more natural trace as the way how the characters have been originally written. We suggest that the manual correction process should be done by a philologist or at least by a person who knows well how to write the ancient characters with a guide of a transcription of the manuscript provided by a philologist.

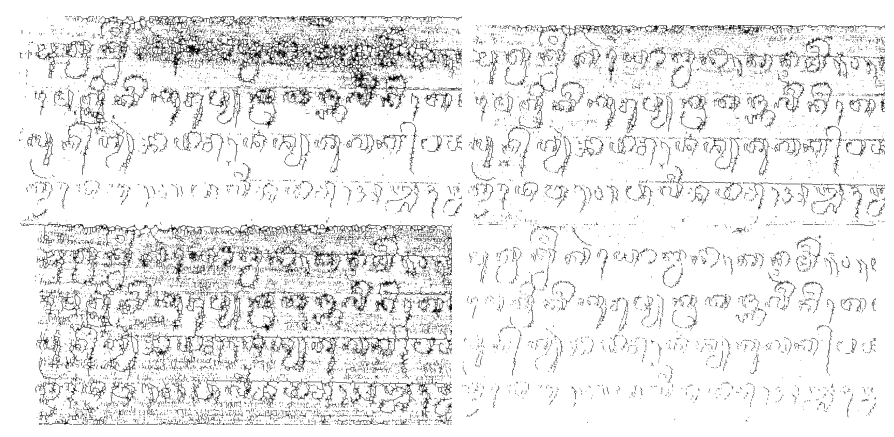

Fig. 2. Examples of image of skeleton (left to right and up to bottom) generated from binarized image of Otsu [8], [9], Niblack [11], Rais [12], and NICK [11]

\section{SPECIFIC BINARIZATION SCHEME PROPOSED}

\section{A. Challenges}

Ancient palm leaf manuscripts contain artefacts due to aging, foxing, yellowing, marks of strain, local shading effects, with low intensity variations or poor contrast, random noises, discoloured part, fading. We can also mention the merges, fractures and other deformations in the character shapes, with the use of nonstandard fonts, varying space between letters, and varying space between lines as illustrated in Fig. 3.

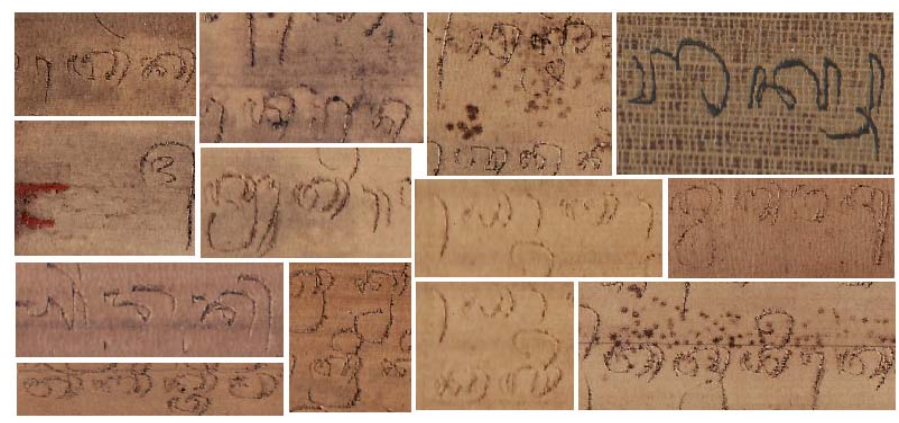

Fig. 3. Samples images of ancient palm leaf manuscripts from Bali

We experimented and compared several alternative wellknown binarization algorithms on our palm leaf manuscript images. Fig. 4 shows the binarized images when applying different method such as Otsu [8][9], Niblack [11]-[15], Sauvola [16], Wolf [11][12], Rais [12], NICK [11], and Howe [17]. Since there is no existing ground truth binarized image for our palm leaf manuscripts, we cannot objectively evaluate these results. Therefore, a visual observation process has been applied to compare the results. It is clear that those binarization methods do not give a good binarized image for palm leaf manuscript images. All methods extract unrecognizable 
characters on palm leaf manuscripts with noise. Howe's method, one of the good ranked methods from DIBCO 2013, does not also provide a good result for this kind of document. Therefore, to binarize the images of palm leaf manuscripts, a specific and adapted binarization technique is required.

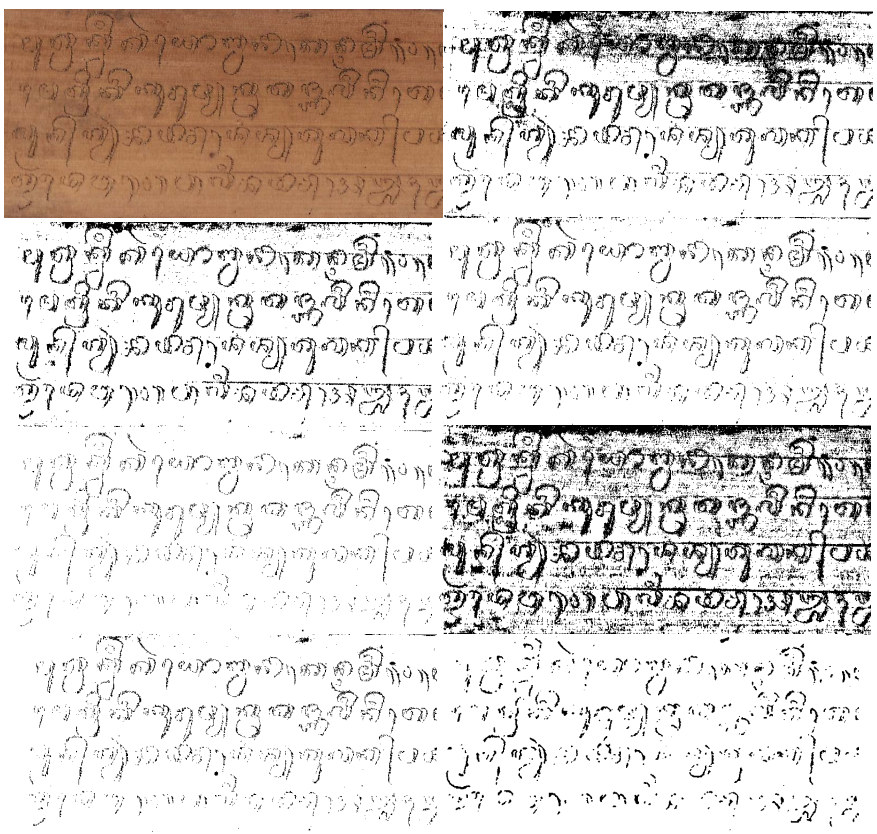

Fig. 4. Original image (upper left), and binarized images (up to bottom,left to right) using methods of Otsu, Niblack, Sauvola, Wolf, Rais, NICK, and Howe

\section{B. Specific scheme for the initial binarization process}

In order to overcome the binarization problem on degraded and low quality palm leaf manuscript image, we proposed a 'semi-local' concept. The idea of this proposed method is to apply a powerful global binarization method on only precise local character area.

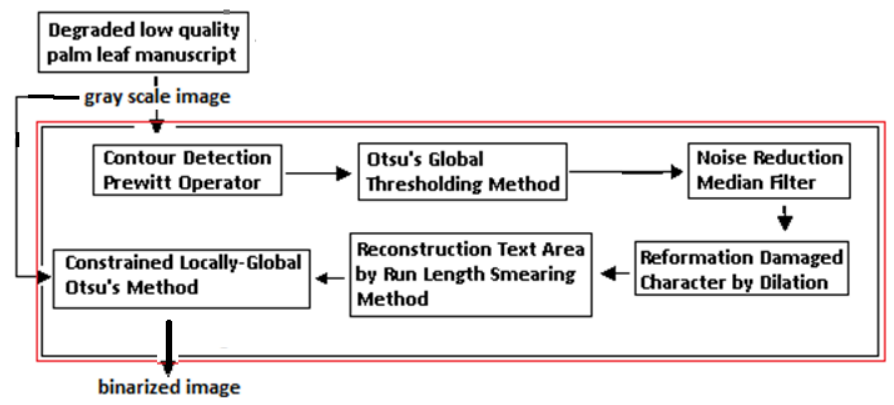

Fig. 5. Semi-local binarization scheme

Our binarization scheme consists of several steps as illustrated in Fig. 5. First, the edge detection with Prewitt Operator is applied to get the initial surrounding area of the line-strokes of each character. Based on our visual observation, Prewitt leads to high edge response on inner part of the characters, and it gives a good approximate area for the skeleton. Whereas Canny leads to high edge response on the outer side of text stroke, and it detects over sensitively the textural part of the palm leaf background. The greyscale image of edge is then binarized with Otsu's method to get the first binarized image of the palm leaf manuscript. Median Filter is then applied to this binarized image in order to remove noise. After noise reduction, some characters might be affected and broken. A dilation process is applied to recover and reform the broken parts of the character. We, then, construct the approximated character area using Run Length Smearing (RLS) method [18]. The smearing method should be done optimally, so the missing/undetected character area can be detected completely. The RLS in row wise will cover the missing area in horizontal strokes character line, meanwhile the RLS in column wise will cover the missing area in vertical strokes character line. After those steps, we finally have a binarized image with an approximated character area in black, and the background area in white. The next step is the main concept of this scheme. We applied Otsu's binarization method for the second time, but locally only within a limited character area, defined by each connected component form the first binarized image generated (Fig. 6).

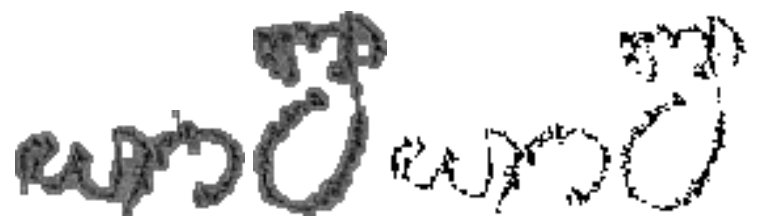

Fig. 6. Examples of extracted character area (on the left) and their semi-local binarization result (on the right).

After the initial binarization process, we can finally perform a morphological-based thinning method to get the skeleton of the character. The thinned image normally still have the unwanted branch, so we apply a morphological-based pruning method to our thinned characters image. A pruning method for the skeleton is effective to remove spurious unwanted parts of the skeleton, and it makes the manual correction process of the skeleton faster. Fig. 7 shows a sample of image sequence as the result of our specific scheme. The goodness of the results can only be estimated qualitatively by examining the results. Based on visual criteria, the proposed scheme provides a good initial image of skeleton with respect to image quality and preservation of meaningful textual character information.

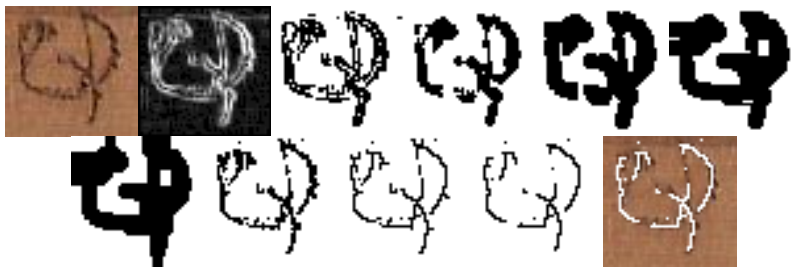

Fig. 7. Original sample image, and sequence sample image of Prewitt, Otsu, Median Filter, Dilation, RLS Row, RLS Col, Local Otsu, Thinning, Pruning, Superposed Skeleton on Original Image

\section{EXPERIMENTS AND RESULTS}

We experimentally tested the framework for construction of ground truth binarized image for nondegraded and degraded low quality palm leaf manucsript images. The sample images for the nondegraded palm leaf manuscript images come from the story of Kakawin Ramayana (reference code 07.41/5634.3 and 07.40/5634.3), in Museum Bali, Denpasar, Indonesia. The palm leaf manuscripts were ordinarily scanned with scanner Epson L210 in 600 dpi without any specific external illumination. The sample images for the degraded low quality palm leaf manuscript images come from the private family collection and Museum Gedong Kertya, Singaraja, Indonesia. The manuscripts were scanned in $300 \mathrm{dpi}$ and it consists of 
several types of degradation as already mentioned in section III.A. The manuscripts were written on both sides, but there was no back-to-front interference observed.

\section{A. Case for nondegraded palm leaf manuscript images}

For nondegraded palm leaf manuscripts, we used a simplest and the most conventional global thresholding method with a proper threshold selected manually to obtain the initial binarized image. With this initial binarized image, it is already sufficient to obtain an acceptable skeletonized image. We performed the manual correction of the skeleton, guided by the transcription of the manuscript provided by a philologist, to finally obtain the skeleton ground truth of the manuscript. Fig. 8 shows a snapshot of simple prototype with user friendly interface that we developed and used to facilitate the manual correction process. We finally constructed the ground truth image by dilating the corrected skeleton image, constrained by the Canny edge image and the initial binarized image from Otsu's global method. We use Otsu's global method instead of the same global fixed thresholding method used in our skeleton ground truth construction because we need a more complete connected component of all characters detected on the binarized image. Other binarization methods can also be used, for example Niblack's method or the multi resolution version of Otsu's method [13]. They also provide a satisfactory preliminary binarized image. Fig. 9 shows an example of final ground truth image from a nondegraded palm leaf manuscript image. It is visually an acceptable estimated ground truth image for the manuscript.

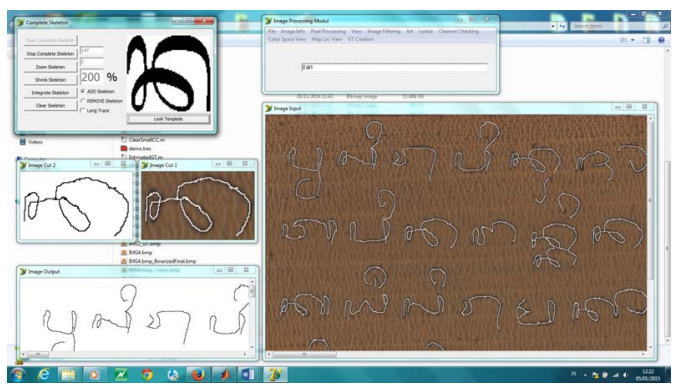

Fig. 8. Snapshot of Prototype Interface used for manual correction of skeleton

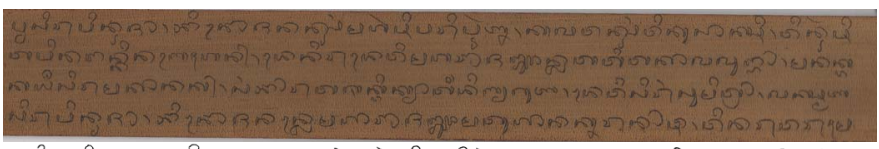

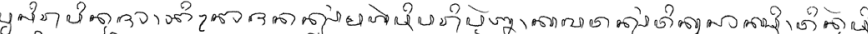

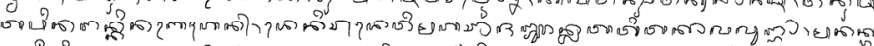

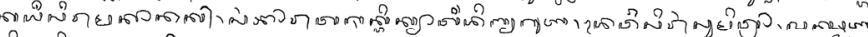

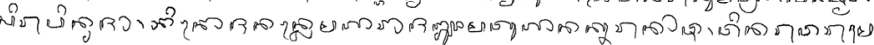

Fig. 9. Estimated ground truth of a nondegraded palm leaf manuscript image

\section{B. Case for degraded low quality palm leaf manuscript images}

For degraded low quality palm leaf manuscript images, we applied our proposed specific binarization scheme by defining the optimal value of parameters based on our empirical experiments as follows: filter size $3 \times 3$ for Median Filter, square structuring element size $3 \times 3$ for Dilation, smearing 3 pixels in row and 3 pixels in column for RLS Method, and pruning the branch of 2 pixels. We performed the manual correction of the skeleton, guided by the transcription of the manuscript provided by a philologist to obtain the skeleton ground truth image of the manuscript. Fig. 10 shows an exemple of a low quality palm leaf manuscript and the skeleton ground truth image. We first experimented the construction of estimated ground truth image by applying a constraint of Canny edge image and an intial binarized image. For example, we used the binarized image from Niblack's method or the multi resolution version of Otsu's method as the constraint. The estimated ground truth image really depends on the initial binarized image used as a constraint. We then experimented the construction of ground truth image without any initial binarized image as a constraint. The result is shown in Fig. 11. Based on visual criteria, the proposed algorithm seems to achieve a better estimated ground truth image with respect to image quality and preservation of meaningful textual character information. Some other results of ground truth binarized image for degraded low quality palm leaf manuscript images are shown in Fig. 12.

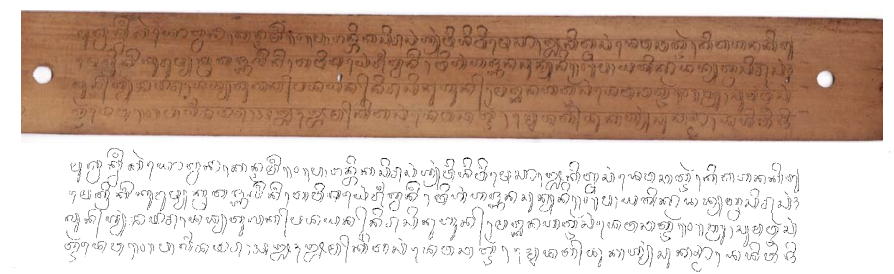

Fig. 10. Original Image and the skeleton ground truth

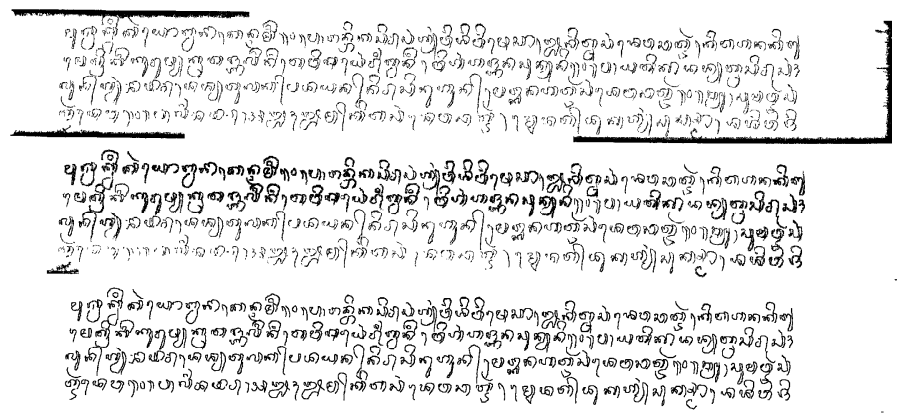

Fig. 11. Ground truth image constructed with an initial binarized image of Niblack's method, Multi Resolution Otsu's method, and without any constraint of initial binarized image

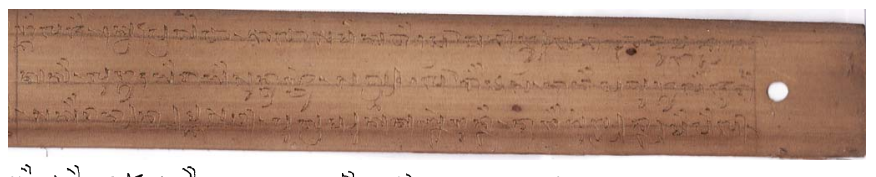

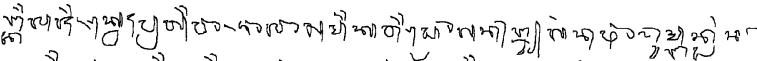

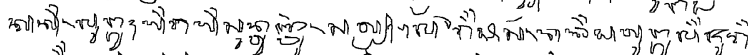

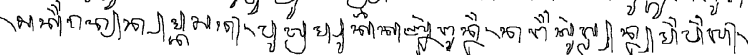
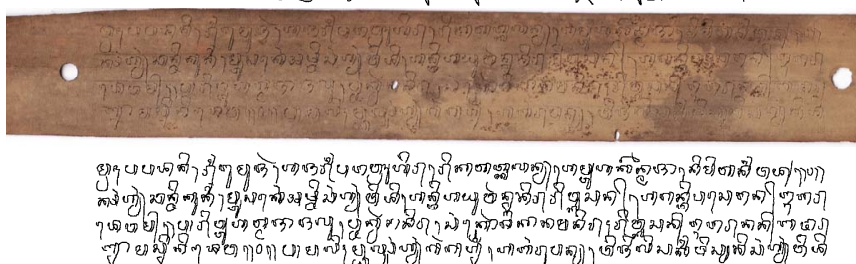

Fig. 12. Two palm leaf manuscript images with their ground truth binarized images

\section{Experiments on the effect of the variation of ground truth image}

Recent studies on the analysis of binarization ground truthing [19] and the effect of ground truth on image binarization [20] discuss the influence human subjectivity during the manual correction process that may produce several different variations of ground truth. We designed an 
experiment to compare the effect of the variation of ground truth image on the performance of the existing binarization methods. We generated two variations of our ground truth binarized image by applying an erosion and a dilation with a simple $3 \times 3$ square structuring element. Those two different versions of our ground truth image represent two possible human subjectivities on the border pixels of the character. We evaluated the performance of the binarization methods by using these three different versions of our ground truth binarized image. Table 1 shows the average value of F-Measure [4] of the binarization method for 9 palm leaf manuscript images. Our experiment shows that the rank performance of the binarization methods evaluated on three different versions of ground truth image is stable. This means that our original ground truth images are robust enough to be used to evaluate and to select future optimal binarizations method for ancient palm leaf manuscripts. The low value of F-Measure for all binarization methods shows that the binarization problem is still an open question especially for specific document images such as our ancient palm leaf manuscript images.

TABEL 1. AVERAge F-MEASURE OF EXISTING BINARIZATION METHOD EVALUATED ON THREE VERSIONS OF GROUND TRUTH IMAGE CREATED BY OUR PROPOSED SCHEME

\begin{tabular}{|c|c|c|c|c|}
\hline Rank & Method & GT Original & GT Eroded & GT Dilated \\
\hline 1 & NICK & 60,48 & 44,78 & 63,47 \\
\hline 2 & SAUVOLA & 57,61 & 40,62 & 61,39 \\
\hline 3 & WOLF & 54,02 & 41,43 & 56,26 \\
\hline 4 & NIBLACK & 48,35 & 29,47 & 54,63 \\
\hline 5 & HOWE & 42,47 & 28,98 & 45,76 \\
\hline 6 & RAIS & 34,61 & 18,82 & 41,68 \\
\hline 7 & OTSU & 32,88 & 18,90 & 41,32 \\
\hline
\end{tabular}

\section{CONCLUSION}

The specific binarization scheme proposed in this paper achieves a better ground truth for degraded low quality palm leaf manuscripts. This scheme adapts and performs better in constructing the ground truth of binarized images for palm leaf manuscripts. The ground truth images constructed with this scheme are robust enough to be used to evaluate and to select future optimal binarization methods for ancient palm leaf manuscripts. The binarization problem is still an open question especially for ancient palm leaf manuscript images.

\section{ACKNOWLEDGMENT}

The authors would like to thank Museum Bali in Denpasar and Museum Gedong Kertya in Singaraja Bali, Indonesia, for providing us the sample of ancient palm leaf manuscripts. This work is also supported by the DIKTI BPPLN Indonesian Scholarship Program and the STIC Asia Program implemented by the French Ministry of Foreign Affairs and International Development (MAEDI).

\section{REFERENCES}

[1] R. Chamchong, C. C. Fung, and K. W. Wong, "Comparing Binarisation Techniques for the Processing of Ancient Manuscripts," in
Cultural Computing, vol. 333, R. Nakatsu, N. Tosa, F. Naghdy, K. W. Wong, and P. Codognet, Eds. Berlin, Heidelberg: Springer Berlin Heidelberg, 2010, pp. 55-64.

[2] Chun Che Fung and R. Chamchong, "A Review of Evaluation of Optimal Binarization Technique for Character Segmentation in Historical Manuscripts," presented at the Third International Conference on Knowledge Discovery and Data Mining, WKDD 2010, 2010, pp. 236-240.

[3] E. Saund, J. Lin, and P. Sarkar, "PixLabeler: User Interface for PixelLevel Labeling of Elements in Document Images," presented at the ICDAR '09. 10th International Conference on Document Analysis and Recognition, 2009, pp. 646-650.

[4] K. Ntirogiannis, B. Gatos, and I. Pratikakis, "An Objective Evaluation Methodology for Document Image Binarization Techniques," in The Eighth IAPR International Workshop on Document Analysis Systems, 2008, 2008, pp. 217-224.

[5] K. Ntirogiannis, B. Gatos, and I. Pratikakis, "Performance Evaluation Methodology for Historical Document Image Binarization," IEEE Trans. Image Process., vol. 22, no. 2, pp. 595-609, Feb. 2013.

[6] H. Z. Nafchi, S. M. Ayatollahi, R. F. Moghaddam, and M. Cheriet, "An Efficient Ground Truthing Tool for Binarization of Historical Manuscripts," presented at the 2013 12th International Conference on Document Analysis and Recognition (ICDAR), 2013, pp. 807-811.

[7] G. Bal, G. Agam, O. Frieder, and G. Frieder, "Interactive degraded document enhancement and ground truth generation," presented at the Proceedings of the SPIE, 2008, vol. 6815, p. 68150Z-68150Z-9.

[8] I. Pratikakis, B. Gatos, and K. Ntirogiannis, "ICDAR 2013 Document Image Binarization Contest (DIBCO 2013)," presented at the ICDAR '13 Proceedings of the 2013 12th International Conference on Document Analysis and Recognition, 2013, pp. 1471-1476.

[9] I. B. Messaoud, H. El Abed, V. Märgner, and H. Amiri, "A design of a preprocessing framework for large database of historical documents," presented at the HIP '11 Proceedings of the 2011 Workshop on Historical Document Imaging and Processing, 2011, p. 177.

[10] J. Canny, "A Computational Approach to Edge Detection," IEEE Trans. Pattern Anal. Mach. Intell., vol. PAMI-8, no. 6, pp. 679-698, Nov. 1986.

[11] K. Khurshid, I. Siddiqi, C. Faure, and N. Vincent, "Comparison of Niblack inspired binarization methods for ancient documents," presented at the Proc. SPIE 7247, Document Recognition and Retrieval XVI, 2009, vol. 7247, p. 72470U-72470U-9.

[12] M. S. H. Naveed Bin Rais, "Adaptive thresholding technique for document image analysis," 2004, pp. 61-66.

[13] M. R. Gupta, N. P. Jacobson, and E. K. Garcia, "OCR binarization and image pre-processing for searching historical documents," Pattern Recognit., vol. 40, no. 2, pp. 389-397, Feb. 2007.

[14] J. He, Q. D. M. Do, A. C. Downton, and J. H. Kim, "A comparison of binarization methods for historical archive documents," in Eighth International Conference on Document Analysis and Recognition, 2005. Proceedings, 2005, pp. 538-542 Vol. 1.

[15] M.-L. Feng and Y.-P. Tan, "Contrast adaptive binarization of low quality document images," IEICE Electron. Express, vol. 1, no. 16, pp. 501-506, 2004.

[16] J. Sauvola and M. Pietikäinen, "Adaptive document image binarization," Pattern Recognit., vol. 33, no. 2, pp. 225-236, février 2000 .

[17] N. R. Howe, "Document binarization with automatic parameter tuning," Int. J. Doc. Anal. Recognit. IJDAR, vol. 16, no. 3, pp. 247258, Sep. 2013.

[18] F. M. Wahl, K. Y. Wong, and R. G. Casey, "Block segmentation and text extraction in mixed text/image documents," Comput. Graph. Image Process., vol. 20, no. 4, pp. 375-390, Dec. 1982.

[19] E. H. B. Smith, "An analysis of binarization ground truthing," presented at the DAS '10 Proceedings of the 9th IAPR International Workshop on Document Analysis Systems, 2010, pp. 27-34.

[20] E. H. B. Smith and C. An, "Effect of 'Ground Truth' on Image Binarization," presented at the DAS '12 Proceedings of the 10th IAPR International Workshop on Document Analysis Systems, 2012, pp. 250-254. 